\title{
ON A PROBLEM OF MAX A. ZORN
}

\section{RIMHAK REE}

1. Introduction. Max A. Zorn has proved ${ }^{1}$ the following theorem.

THEOREM. If every substitution $x=a t, y=b t$ in which $a$ and $b$ are complex numbers transforms $\sum a_{i j} x^{i} y^{j}$ into a power series with a nonvanishing radius of convergence, the series $\sum\left|a_{i j} x^{i} y^{j}\right|$ converges for sufficiently small $|x|$ and $|y|$.

He has also suggested the following problem. If $\sum a_{i j} x^{i} y^{j}$ is a power series which is transformed by every substitution of convergent power series $\sum_{1}^{\infty} a_{i} t^{i}$ and $\sum_{1}^{\infty} b_{i} t^{i}$ with real coefficients for $x$ and $y$ into a convergent power series in $t$, is the double series $\sum a_{i j} x^{i} y^{j}$ convergent?

The answer is yes. In fact, Zorn's theorem itself holds even when the coefficients $a$ and $b$ are restricted to take only real values. We can obtain a proof quite directly by Zorn's method, if we use an estimate for the coefficients of homogeneous polynomials in real variables.

2. Homogeneous polynomials in real variables. We shall prove a lemma which may easily be extended to the case of many variables.

Lemma. Let $P(x, y)=\sum_{i+j=n} a_{i j} x^{i} y^{j}$ be a homogeneous polynomial in real variables. If $|P(x, y)| \leqq M$ for $\left|x-x_{0}\right| \leqq 2 \delta,\left|y-y_{0}\right| \leqq 2 \epsilon$, then $\left|a_{i j} \delta^{i} \epsilon^{j}\right| \leqq M$.

Proof. Set $x=x_{0}+\delta\left(\xi+\xi^{-1}\right)$. Then $\xi^{n} P(x, y)=\sum a_{i j} \xi^{j}(\xi x)^{i} y^{j}$ is a polynomial in $\xi$ whose absolute value does not exceed $M$ when $\xi$ moves on the unit circle of the Gaussian plane. By Cauchy's inequality of function theory, and considering the coefficients of $\xi^{k}$ in $\xi^{n} P(x, y)$, we have

$$
\left|\sum_{j=0}^{k} a_{i j} c_{i} y^{j}\right| \leqq M,
$$

where $0 \leqq k \leqq n, i+j=n$, and $c_{i}$ is the coefficient of $\xi^{k-j}$ in $(\xi x)^{i}$.

Again set $y=y_{0}+\epsilon\left(\eta+\eta^{-1}\right)$ and apply the Cauchy inequality to the constant term of $\eta^{k} \sum_{j=0}^{k} a_{i j} c_{i} y^{j}$. We have

$$
\left|a_{l k} c_{l} \epsilon^{k}\right| \leqq M
$$

where $l+k=n$ and $c_{l}$ equals $\delta^{l}$ by definition. This completes our proof.

Received by the editors May 10, 1948 .

1 Bull. Amer. Math. Soc. vol. 53 (1947) pp. 791-792. 
3. Proof of Zorn's theorem in the real case. Now we can follow Zorn's method directly.

Proof. Let $P_{n}(x, y)=\sum_{i+j=n} a_{i j} x^{i} y^{j}$. The set $D$ of vectors $(x, y)$ for which $\sum P_{n}(x, y)$ converges is of the second category. For every vector is in $^{2} m D$ for some positive integer $m$. If $D$ were of the first category, the set $m D$ and therefore the two-dimensional Euclidean space would be the same character.

By virtue of the continuity of the functions $P_{n}$ there will exist a square $\left|x-x_{0}\right| \leqq 2 p,\left|y-y_{0}\right| \leqq 2 p, \quad p>0$ and an $M$ such that $\left|P_{n}(x, y)\right| \leqq M$ holds in the square for all $n$. Then by our lemma $\left|a_{i j} p^{i+j}\right| \leqq M$, Hence for $|x|,|y| \leqq p / 2$, we have

$$
\left|a_{i j} x^{i} y^{i}\right| \leqq M / 2^{i+j}
$$

which establishes the absolute convergence of the double series.

SEOUL University, KoreA

${ }^{2} m D$ is the set of $(m x, m y)$ where $(x, y) \in D$. 